\title{
Fabrication of SERS Active Surface on Polyimide Sample by Excimer Laser Irradiation
}

\author{
T. Csizmadia, ${ }^{1}$ B. Hopp, ${ }^{1}$ T. Smausz, ${ }^{2}$ Zs. Bengery, ${ }^{1}$ J. Kopniczky, ${ }^{1}$ \\ I. Hanyecz, ${ }^{1}$ and G. Szabó ${ }^{1,2}$ \\ ${ }^{1}$ Department of Optics and Quantum Electronics, University of Szeged, Dóm tér 9 Szeged 6720, Hungary \\ ${ }^{2}$ MTA-SZTE Research Group on Photoacoustic Spectroscopy, University of Szeged, Dóm tér 9 Szeged 6720, Hungary
}

Correspondence should be addressed to T. Csizmadia; csizmadia87@gmail.com

Received 16 October 2013; Accepted 31 January 2014; Published 2 March 2014

Academic Editor: Denis Chaumont

Copyright (C) 2014 T. Csizmadia et al. This is an open access article distributed under the Creative Commons Attribution License, which permits unrestricted use, distribution, and reproduction in any medium, provided the original work is properly cited.

\begin{abstract}
A possible application of excimer laser irradiation for the preparation of surface enhanced Raman spectroscopy (SERS) substrate is demonstrated. A polyimide foil of $125 \mu \mathrm{m}$ thickness was irradiated by 240 pulses of focused ArF excimer laser beam $(\lambda=193 \mathrm{~nm}$, FWHM $=20 \mathrm{~ns}$ ). The applied fluence was varied between 40 and $80 \mathrm{~mJ} / \mathrm{cm}^{2}$. After laser processing, the sample was coated with $40 \mathrm{~nm}$ silver by PLD in order to create a conducting layer required for the SERS application. The SERS activity of the samples was tested by Raman microscopy. The Raman spectra of Rhodamine $6 \mathrm{G}$ aqueous solution $\left(\mathrm{c}=10^{-3} \mathrm{~mol} / \mathrm{dm}^{3}\right)$ were collected from the patterned and metalized areas. For areas prepared at $40-60 \mathrm{~mJ} / \mathrm{cm}^{2}$ laser fluences, the measured Raman intensities have shown a linear dependence on the applied laser fluence, while above $60 \mathrm{~mJ} / \mathrm{cm}^{2}$ saturation was observed. The morphology of the SERS active surface areas was investigated by scanning electron microscopy. Finite element modeling was performed in order to simulate the laser-absorption induced heating of the polyimide foil. The simulation resulted in the temporal and spatial distribution of the estimated temperature in the irradiated polyimide sample, which are important for understanding the structure formation process.
\end{abstract}

\section{Introduction}

Raman spectroscopy (RS) is one of the most common spectroscopic methods for studying the vibrational and rotational energy levels of an analyte [1]. Compared to other spectroscopic techniques, RS is fast and nondestructive and needs practically no sample preparation and its microscope/imaging version has very good lateral resolution (about $1-2 \mu \mathrm{m}$ ). However, since only a very small fraction of the incident photons are Raman scattered (in general, the intensity of the Raman signal is orders of magnitude weaker than the elastic scattering intensity) [2], the range of detectable molecules and concentrations is significantly reduced. For that reason, enhancing the Raman signal produced by molecules was always in the focus of attention.

A significant increase of the Raman signal can be achieved when the target molecules are absorbed on adequately structured conducting surfaces. According to theory [3], surface enhanced Raman scattering (SERS) can be attributed to the laser-induced excitation of surface plasmons, resulting in the increase of the local electric field above the surface. The Raman intensities strongly depend on the magnitude of the developed electric near field, and thereby high enhancement can occur in the measured signal (up to $10^{10}$ in certain cases) [4].

One of the most widely used SERS active substrates is metal colloids (especially aggregated colloids) produced by chemical methods [5-8]. Another study aimed at the production of appropriate composite arrangement, where silver colloids were prepared by adding silver nitrate to the aqueous solution of excess sodium borohydride, the reducing agent. The silver colloids were then deposited on smooth silver, copper, and nickel plates. The composite structure was obtained by inserting a monolayer or submonolayer of organic molecules between two of these silver-colloid-covered metal substrates in a sandwich arrangement. On this way the analyte molecules are adsorbed onto the metal plates as ligands and are also in close contact with the silver nanoparticles, which ensure the enhancement for the Raman spectra of the adsorbates [9]. 
In another experiment, pulsed laser ablation (PLA) was used to fabricate silver chloride $(\mathrm{AgCl})$ nanocubes directly from a bulk target of silver in sodium chloride $(\mathrm{NaCl})$ solution. The size of the particles was optimized and the surface properties of the cubes were investigated for their SERS activity for Rhodamine $6 \mathrm{G}$ (R6G). The results indicated that the $\mathrm{AgCl}$ nanocubes obtained using PLA could largely enhance the Raman signal of R6G molecules, which makes them a useful substrate for practical SERS measurements [10].

In our previous study, the applicability of the laserinduced backside dry etching (LIBDE) technique was demonstrated to form such nanostructures on the surface of a fused silica plate, which are able to produce the SERS effect, when they are subsequently coated with a conducting layer. This method proved to be a relatively cheap and promising technique in the production of SERS substrates without the necessity of specific toolkits; however, the reproducibility of the most suitable surface structures and the size of the nanostructured area on the surface need to be improved for practical use [11].

In this paper the preparation of nanostructures by excimer laser irradiation of polyimide foil is presented and the suitability of the produced morphologies in creating the surface enhanced Raman scattering effect is demonstrated. Our motivation was to produce a relatively large and homogeneous surface with remarkable SERS activity and good reproducibility by the use of a simple laser-based micromachining technique and cheap substrate material.

\section{Experiments}

Kapton $\mathrm{HN}$ polyimide foil of $125 \mu \mathrm{m}$ thickness was irradiated by 240 pulses of focused ArF excimer laser beam $(\lambda=$ $193 \mathrm{~nm}, \mathrm{FWHM}=20 \mathrm{~ns}$ ). The penetration depth at this laser wavelength is as low as $23 \mathrm{~nm}$ due to the high absorption coefficient of polyimide $\left(\alpha=43 \mu \mathrm{m}^{-1} @ 193 \mathrm{~nm}\right)$ [12]. The illuminated area was $7.07 \mathrm{~mm}^{2}$ and the applied fluence was varied between 40 and $80 \mathrm{~mJ} / \mathrm{cm}^{2}$, which was verified by an out-coupled reference beam during the irradiations. After each series of pulses, the sample was moved by an $x-y$ translator, in order to ensure an intact surface area for the next series. Due to the laser treatment, the irradiated surface darkened, which was more discernible in the lower part of the applied fluence range. Previously, Ball et al. observed the decrease in the reflectivity of polyimide due to the formation of carbon clusters on the excimer laser illuminated polymer surface $[13,14]$.

Although former experiments showed that the electrical conductivity of the polyimide surface increases significantly in response to the excimer laser irradiation [13-15], our attempts to use these solely laser treated surfaces as SERS substrates were unsuccessful. In order to insure the sufficient conducting surface for SERS, the laser patterned surfaces were coated with a thin layer of silver produced by pulsed laser deposition (PLD). The PLD process was carried out in a vacuum chamber at a pressure of approximately $4.5 \cdot 10^{-3} \mathrm{~Pa}$, by using the same ArF excimer laser applied for surface structuring. In this case the laser beam was focused onto a silver target at an incident angle of $45^{\circ}$, which generated a spot size of $4.24 \mathrm{~mm}^{2}$ and an average fluence of $570 \mathrm{~mJ} / \mathrm{cm}^{2}$. The target sample was shot by 15000 laser pulses and the ejected silver clouds were deposited onto the polyimide surface placed $3 \mathrm{~cm}$ directly in front of the silver target. The thickness of the produced silver film was $40 \mathrm{~nm}$ on the average, which was measured by a Dektak 8 surface profiler after the PLD process.

\section{Results and Discussion}

After the deposition of the thin conducting metal layer, SERS activity of the laser-irradiated and silver covered polyimide surface areas was tested by a Thermo Scientific DXR Raman microscope, operated at laser wavelength of $780 \mathrm{~nm}$ and laser power of $5 \mathrm{~mW}$ using a 10x objective and a $50 \mu \mathrm{m}$ slit. $10^{-3} \mathrm{~mol} / \mathrm{dm}^{3}$ aqueous solution of Rhodamine $6 \mathrm{G}$ (R6G) dye was used as test sample. The polyimide foil was fixed on the bottom of a Petri dish and the dye solution was streamed to the dish until it just covered the sample. The thickness of the R6G solution above the sample was few hundreds of micrometers, which was high enough to consider the concentration of R6G to be constant during the Raman measurements, which took a few minutes. Optical microscope images taken from the excimer laser treated sample exhibited circular spots of about $3 \mathrm{~mm}$ in diameter, corresponding to the irradiated areas. Since the irradiation experiments were carried out around the ablation and carbonization threshold fluence values, the laser-induced changes were very sensitive for the inhomogeneities of the excimer laser beam intensity. This could be seen in the morphological inhomogeneities of the resulting spots as well. In each laser spot three specific (bright, gray, and dark) terrains could be distinguished. The Raman exciting laser beam was focused in a few $\mu \mathrm{m}$ sized spot onto the studied surface of a given laser treated area, and at least four Raman spectra were recorded on every specific terrain type. The characteristic Raman shift peaks of polyimide did not appear in the Raman spectra during SERS investigations, since the transmittance of the applied silver film was less than $4 \%$ [16], which means that only a very small portion of the intensity of the Raman laser light reached the surface of the polyimide sample and an even smaller amount of the scattered light left it, which resulted in the disappearance of the characteristic polyimide peaks observed during reference measurements on the uncoated samples. The characteristic Raman shift peaks of the R6G at $611,773,1362$, and $1510 \mathrm{~cm}^{-1}$ [17] were identified in every recorded spectrum. On the bright and dark terrains, there was no significant SERS enhancement; nevertheless on the gray shaded places strong increases were observed in the intensity of the characteristic peaks with about $13 \%$ standard deviations.

Figure 1 shows the dependence of the measured Raman intensities on the applied excimer laser fluence in case of the SERS active terrains of the irradiated spots at the four wavenumbers of the selected peaks in the R6G spectrum. It can be seen that a higher patterning fluence results in higher Raman intensities in the investigated range. Between 40 and $60 \mathrm{~mJ} / \mathrm{cm}^{2}$, the tendency of the measured Raman intensity 


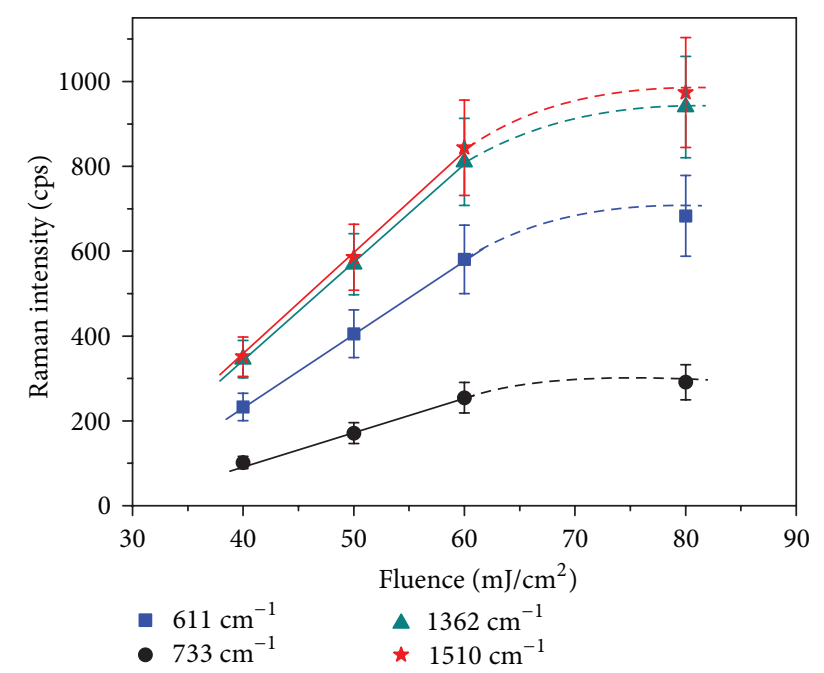

Figure 1: The average Raman intensity set against the applied patterning laser fluence in case of the most SERS active terrains of the irradiated spots at the typical peaks of the R6G Raman spectrum.

curves can be fairly approximated with a linear function, while, above $60 \mathrm{~mJ} / \mathrm{cm}^{2}$, saturation can be observed.

The morphology of the sample surface was investigated by a Hitachi S4700 scanning electron microscope (SEM) in order to study the nanometer-sized structures, which can be relevant in causing the SERS effect. In order to prevent the investigated surface from charging or electron beam induced modification, a relatively low, $1 \mathrm{kV}$ accelerating voltage and $4 \mu \mathrm{A}$ emission current were used without the application of any additional conductive coating (in addition to the PLD deposited silver thin film), which would have covered the existing nanostructures.

Figure 2 shows the low magnification images of the silver coated surface areas of polyimide irradiated at different fluences. Three characteristic terrains can be distinguished macroscopically in almost each etched hole: dark, gray, and bright terrains, which are marked with green (1), blue (2), and red (3) fills and outlines, respectively, in Figure 2. These separated terrain types are equivalent to those, which were identified previously on the optical microscope image of the Raman system. For all fluences, the gray part (marked with green and (1) in Figure 2)-where significant Raman enhancements could be observed-covers at least half of the total irradiated area. This means that the ratio of this SERS useful area compared to the total irradiated area is at least twice more than it was for the etched holes prepared by LIBDE in our former experiments [11].

Figure 3 represents high magnification SEM images of the SERS active surfaces, where conical-like structures with variable size can be noticed typically. The average size of the cones increased with increasing fluence, and also the conical shapes were more defined as it was also revealed previously by Hopp et al. [18]. Between the conical structures, the surface is densely covered by irregular debris for all irradiation fluences (Figure 4). It is discernible from Figure 4 that at higher fluences the surface coverage by debris-like structures is higher, and a more expressed fine structure can be seen overall. Considering the small standard deviations experienced in the SERS enhancements at various places of the gray terrain, it is likely that rather these finely structured terrains having extensive coverage on the irradiated surface are responsible for the generation of the SERS effect than the sparsely located conical structures.

Energy dispersive X-ray spectroscopy (EDX) measurements revealed that the proportion of carbon atoms increased inside the laser processed terrains: $5.5 \%$ increment was found in the gray terrains and $9.1 \%$ in the dark ones on the average. This indicates that the generated surface structures may not only consist of pure polyimide, but carbon clusters and polyimide fragments as well, which was proved by Chtaib et al. in earlier XPS measurements on ArF excimer laser irradiated polyimide [19]. It is known that in case of polyimide at temperatures higher than $793 \mathrm{~K}$ a chemical decomposition occurs instead of melting or evaporation [20]. Our observations imply that high temperature induced surface patterning of polyimide is a criterion for its SERS activity, which needs sufficiently high temperature provided by the absorption of the applied laser energy. In order to model this process, the spatial and temporal temperature distribution in the irradiated polyimide foil was calculated by finite element modeling, using the Comsol Multiphysics package. The absorbed laser energy in the polyimide was considered to be a heat source, which has a Gaussian temporal profile and an exponential spatial (depth) profile according to the Beer-Lambert absorption law. The results showed that the temperature of the irradiated foil exceeds the decomposition temperature of the polyimide down to $140 \mathrm{~nm}$ depth after one laser pulse of $40 \mathrm{~mJ} / \mathrm{cm}^{2}$ fluence, which explains the chemical modifications on the irradiated surface observed by EDX. Furthermore, ablation occurs in the whole applied fluence range, since the applied fluence was above the ablation threshold fluence $\left(\sim 25 \mathrm{~mJ} / \mathrm{cm}^{2}\right.$ for polyimide at $193 \mathrm{~nm}$ [21]). The ablation process is important regarding the mechanism of the surface structure formation. It has been observed earlier that direct etching that is ablation of polyimide can result in microstructures having well-defined shapes [18, 22, 23], which are conical in case of our experimental conditions [18]. However, our results indicate that in the SERS point of view not the conical microstructures are responsible for activating the SERS effect, but the ablated polyimide micro- and nanofragments, which are backscattered from the plume and redeposited onto the irradiated polymer surface, have a substantial role. This effect is similar to the inverse PLD, discovered by Égerházi et al. [24]. Not only the fine structure but also the quantity of the ablated and redeposited material is a significant parameter concerning the SERS activity of the surface. In order to find out the role of these we measured the etch depths of the ablated holes by a Dektak 8 surface profiler. Knowing the irradiated area and the depths of the etched holes, we could give an upper estimation for the ablated volume of the ablated material as a function of the applied laser fluence (Figure 5). The calculated volume of the removed material during excimer laser ablation and the measured Raman intensity shows similar tendencies as 


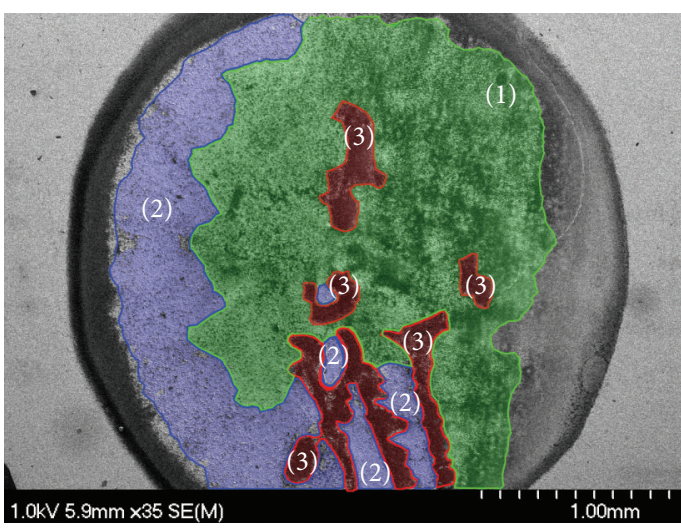

(a)

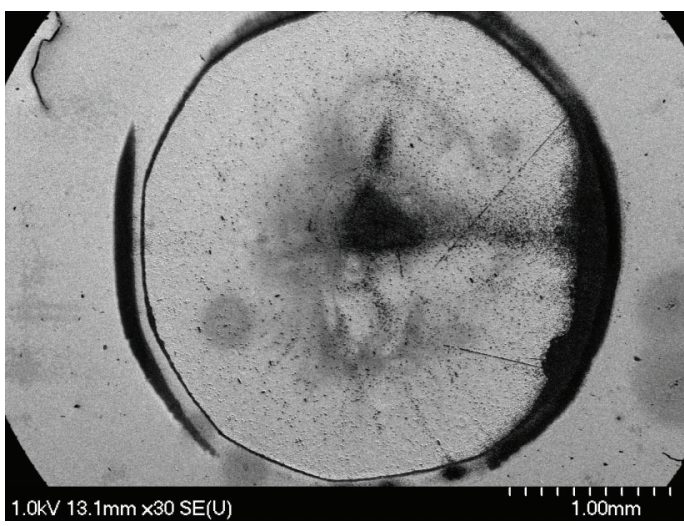

(c)

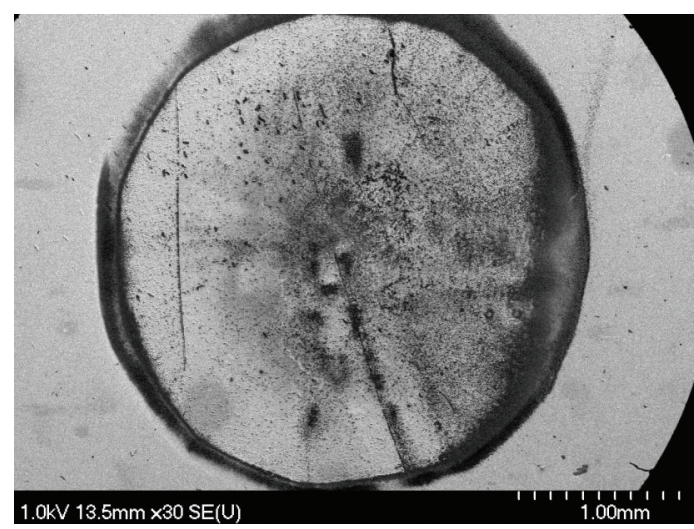

(b)

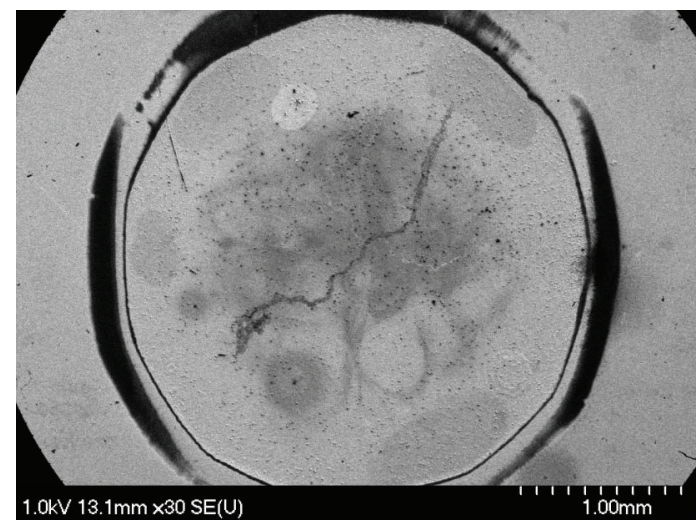

(d)

FIGURE 2: Low magnification SEM pictures on the laser treated spots produced by different excimer laser fluences: 40 (a); 50 (b); 60 (c); and $80 \mathrm{~mJ} / \mathrm{cm}^{2}$ (d). The marked numbered areas indicate the three distinguishable terrain types.

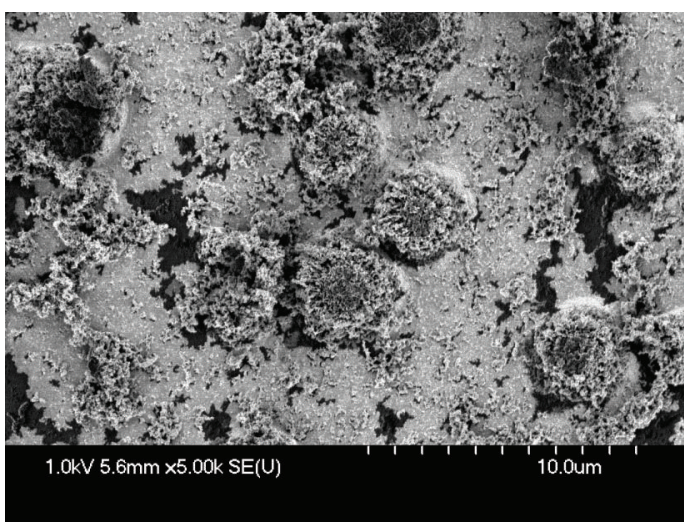

(a)

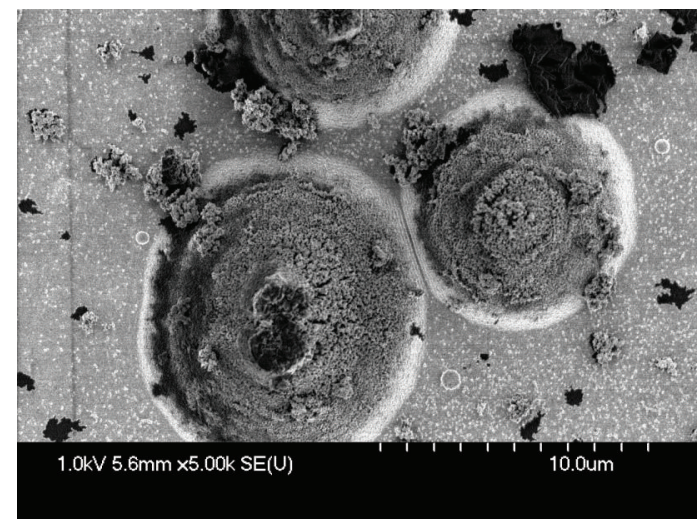

(b)

FIGURE 3: High magnification (5000x) SEM images on the SERS active surfaces prepared at 40 (a) and $80(\mathrm{~b}) \mathrm{mJ} / \mathrm{cm}^{2} \mathrm{fluences}$.

functions of fluence (Figure 1) indicating that there might be a correlation between these two separate processes.

Regarding the mechanism of the SERS activity of the system, it is known that silver layers having approximately $40 \mathrm{~nm}$ thickness support plasmon resonances [25]. Besides that, it is plausible that the enhancement of the Rhodamine $6 \mathrm{G}$ peaks on nanostructured silver surface may not only result from an enhancement in the electric field provided by the surface, but the interaction of chemisorbed R6G molecules with ballistic electrons that are generated through plasmon 


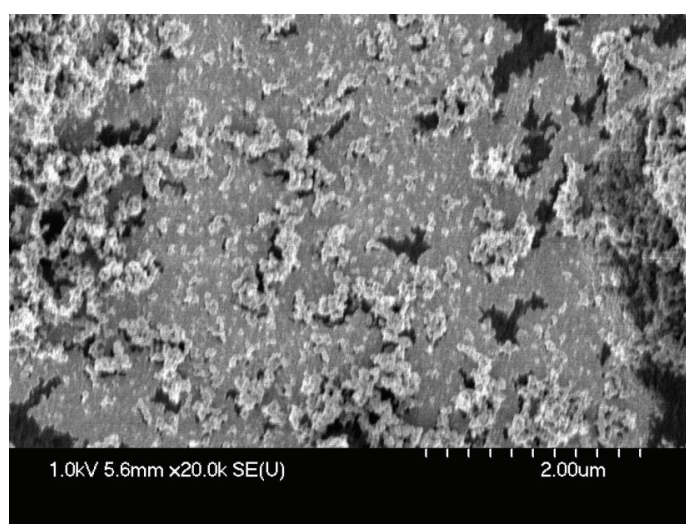

(a)

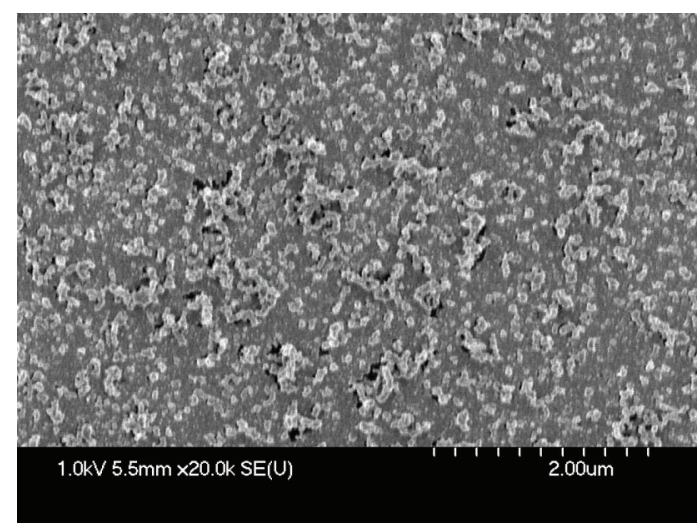

(b)

FIGURE 4: 20 000x magnification images on the fine structures of two SERS active surface areas fabricated at 40 (a) and $80(\mathrm{~b}) \mathrm{mJ} / \mathrm{cm}^{2} \mathrm{fluences}$.

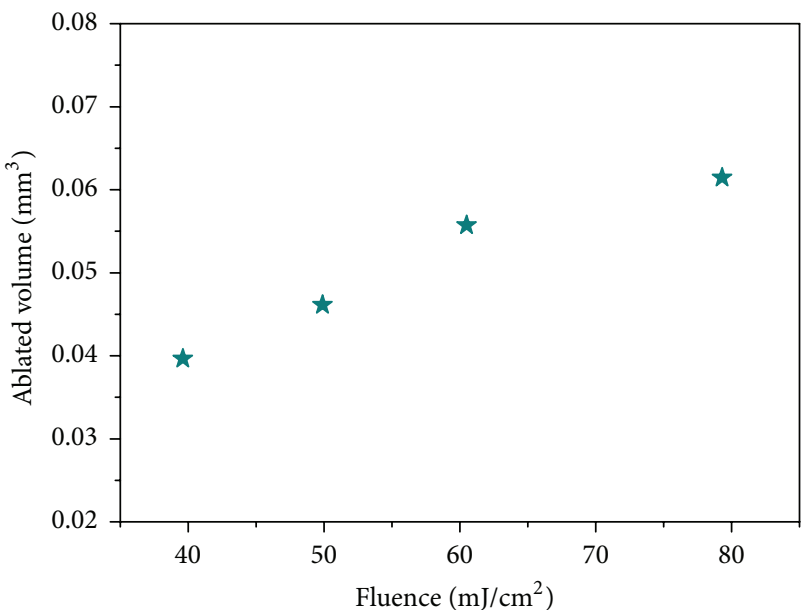

FIGURE 5: The dependence of the etch depth on the applied laser fluence during polyimide ablation.

excitation. A detailed discussion of such mechanism was presented by Michaels et al. regarding single molecule SERS of Rhodamine 6G in the presence of silver nanoparticles [26].

\section{Conclusions}

The preparation of micro- and nanostructures by excimer laser irradiation of polyimide was presented for the purpose of manufacturing a substrate for surface enhanced Raman scattering applications. In order to reach the necessary conductivity of the surface for SERS, the laser treated polyimide foil was covered by a thin layer of silver by PLD. Three types of typical structures could be discerned in each irradiated spot: dark, gray, and bright terrains regarding the optical microscopic investigations and low-magnification SEM images. Considerable SERS intensity enhancement was observed on the gray parts of the irradiated areas compared to the unirradiated and also metal coated surface areas. We found that the SERS activity of the irradiated areas prepared at higher laser fluences was higher. By taking a closer look at the gray parts of the irradiated spots by SEM and recording EDX spectra at the same places, we observed that besides conical microstructures the surface morphology is finely structured, and indications for chemical decomposition (carbonization) can be observed. Our model calculations for spatial and temporal temperature distribution in the irradiated polyimide foil have proved that the required conditions are fulfilled not only for chemical decomposition but also for ablation. Therefore we presume that redeposition of microand nanofragments backscattered from the plume during the ablation process resulted in the observed fine structure of the SERS active areas.

As a conclusion, the applied polyimide is a cheap material for SERS substrate purposes, while nanosecond excimer laser irradiation is a fast production method without the need of any specific toolkits excepting the irradiating laser source. Regarding the structured surface of the created substrate, the size of the SERS active area covered at least half of the total irradiated area in case of every fluence values, which is more than 7 times larger in absolute value than the most enhancing area in our former experiments, where the SERS substrate was prepared by LIBDE, which is a slightly complicated method for material fabrication [11]. In addition, considering the 13\% standard deviation in the measured Raman intensities of the characteristic peaks of the R6G, the reproducibility of the produced structures was good.

\section{Conflict of Interests}

The authors declare that there is no conflict of interests regarding the publication of this paper.

\section{Acknowledgments}

This work was partially supported by the European Union and cofinanced by the European Social Fund through projects Impulse Lasers for Use in Materials Science and Biophotonics (Grant no. TÁMOP-4.2.2.A-11/1/KONV-2012-0060) and Supercomputer, the National Virtual Lab (TÁMOP-4.2.2.C$11 / 1 / K O N V-2012-0010)$ and was realized in the frames of 
National Excellence Program-Elaborating and Operating an Inland Student and Researcher Personal Support System Convergence program (TÁMOP 4.2.4. A/2-11-1-2012-0001).

\section{References}

[1] E. Smith and G. Dent, Modern Raman Spectroscopy-A Practical Approach, John Wiley \& Sons, Hoboken, NJ, USA, 2005.

[2] D. W. Hahn, "Raman Scattering Theory," 2014, http://plaza.ufl .edu/dwhahn/Raman\%20Scattering\%20Theory.pdf.

[3] M. Moskovits, "Surface-enhanced Raman spectroscopy: a brief retrospective," Journal of Raman Spectroscopy, vol. 36, no. 6-7, pp. 485-496, 2005.

[4] P. G. Etchegoin and E. C. le Ru, Basic Electromagnetic Theory of SERS, Surface Enhanced Raman Spectroscopy: Analytical, Biophysical and Life Science Applications, Wiley-VCH, Weinheim, Germany, 2011.

[5] A. Débarre, R. Jaffiol, C. Julien, P. Tchénio, and M. Mostafavi, "Raman scattering from single Ag aggregates in presence of EDTA," Chemical Physics Letters, vol. 386, no. 4-6, pp. 244-247, 2004.

[6] M. Z. Si, Y. P. Kang, and Z. G. Zhang, "Surface-enhanced Raman scattering (SERS) spectra of Methyl Orange in Ag colloids prepared by electrolysis method," Applied Surface Science, vol. 255, no. 11, pp. 6007-6010, 2009.

[7] J. G. S. Lopes and P. S. Santos, "The SERS spectrum of 1,2diaminoanthraquinone studied on silver colloid," Journal of Molecular Structure, vol. 744-747, pp. 75-78, 2005.

[8] M. Muniz-Miranda, N. Neto, and G. Sbrana, "Surface studies by SERS and SEM techniques on filters coated with colloidal silver," Journal of Molecular Structure, vol. 410-411, pp. 205-208, 1997.

[9] M. Muniz-Miranda, B. Pergolese, A. Bigotto, A. Giusti, and M. Innocenti, "A Raman and AFM investigation on metal surfaces SERS-activated by silver colloidal nanoparticles," Materials Science and Engineering C, vol. 27, no. 5-8, pp. 1295-1299, 2007.

[10] C. Dong, Z. Yan, J. Kokx, D. B. Chrisey, and C. Z. Dinu, "Antibacterial and surface-enhanced Raman scattering (SERS) activities of $\mathrm{AgCl}$ cubes synthesized by pulsed laser ablation in liquid," Applied Surface Science, vol. 258, pp. 9218-9222, 2012.

[11] T. Csizmadia, B. Hopp, T. Smausz et al., "Possible application of laser-induced backside dry etching technique for fabrication of SERS substrate surfaces," Applied Surface Science, vol. 278, pp. 234-240, 2013.

[12] H. R. Philipp, H. S. Cole, Y. S. Liu, and T. A. Sitnik, "Optical absorption of some polymers in the region 240-170 nm," Applied Physics Letters, vol. 48, no. 2, pp. 192-194, 1986.

[13] Z. Ball, B. Hopp, M. Csete et al., "Transient optical properties of excimer-laser-irradiated polyimide-I. Refractive index," Applied Physics A, vol. 61, no. 5, pp. 547-551, 1995.

[14] Z. Ball, B. Hopp, M. Csete et al., "Transient optical properties of excimer-laser-irradiated polyimide-II. Carbon-cluster scattering," Applied Physics A, vol. 61, no. 6, pp. 575-578, 1995.

[15] B. Hopp, Z. Szilassi, K. Révész, Z. Kocsis, and I. Mudra, "Excimer laser induced conductivity on silver salt filled polyimide films," Applied Surface Science, vol. 109-110, pp. 212-217, 1997.

[16] http://refractiveindex.info, 2014.

[17] X. Hou, X. Zhang, S. Chen, H. Kang, and W. Tan, "Facile synthesis of $\mathrm{Ni} / \mathrm{Au}, \mathrm{Ni} / \mathrm{Ag}$ hybrid magnetic nanoparticles: new active substrates for surface enhanced Raman scattering," Colloids and Surfaces A, vol. 403, pp. 148-154, 2012.
[18] B. Hopp, Zs. Bor, E. Homolya, and E. Mihalik, "Conical structures produced by excimer laser irradiaton of polymers," in GR-I International Conference on New Laser Technologies and Applications, vol. 3423 of Proceedings of the SPIE, pp. 389-393, 1997.

[19] M. Chtaïb, E. M. Roberfroid, Y. Novis, J.-J. Pireaux, and R. Caudano, "Polyimide surface degradation: $\mathrm{x}$-ray photoelectron spectroscopic study under UV-pulsed laser irradiation," Metallization of Polymers, vol. 11, pp. 161-169, 1990.

[20] Dupont Kapton Polyimide Film General Specifications, Bulletin GS-96-7, 2014, http://www2.dupont.com/Kapton/en_US/ assets/downloads/pdf/Gen_Specs.pdf.

[21] M. Kahrizi, "Laser ablation for polymer waveguide fabrication," in Micromachining Techniques for Fabrication of Micro and Nano Structures, S. Zakariyah, Ed., InTech, Rijeka, Hungary, 2012.

[22] H. Y. Zheng, T. T. Tan, and W. Zhou, "Studies of KrF laserinduced long periodic structures on polyimide," Optics and Lasers in Engineering, vol. 47, no. 1, pp. 180-185, 2009.

[23] B. T. Least and D. A. Willis, "Modification of polyimide wetting properties by laser ablated conical microstructures," Applied Surface Science, vol. 273, pp. 1-11, 2013.

[24] L. Égerházi, Zs. Geretovszky, T. Csákó, and T. Szörényi, “Atomic force microscopic characterization of films grown by inverse pulsed laser deposition," Applied Surface Science, vol. 252, pp. 4661-4666, 2006.

[25] S. K. Cushing, L. A. Hornak, J. Lankford, Y. Liu, and N. Wu, "Origin of localized surface plasmon resonances in thin silver film over nanosphere patterns," Applied Physics A, vol. 103, no. 4, pp. 955-958, 2011.

[26] A. M. Michaels, M. Nirmal, and L. E. Brus, "Surface enhanced Raman spectroscopy of individual rhodamine $6 \mathrm{G}$ molecules on large Ag nanocrystals," Journal of the American Chemical Society, vol. 121, no. 43, pp. 9932-9939, 1999. 

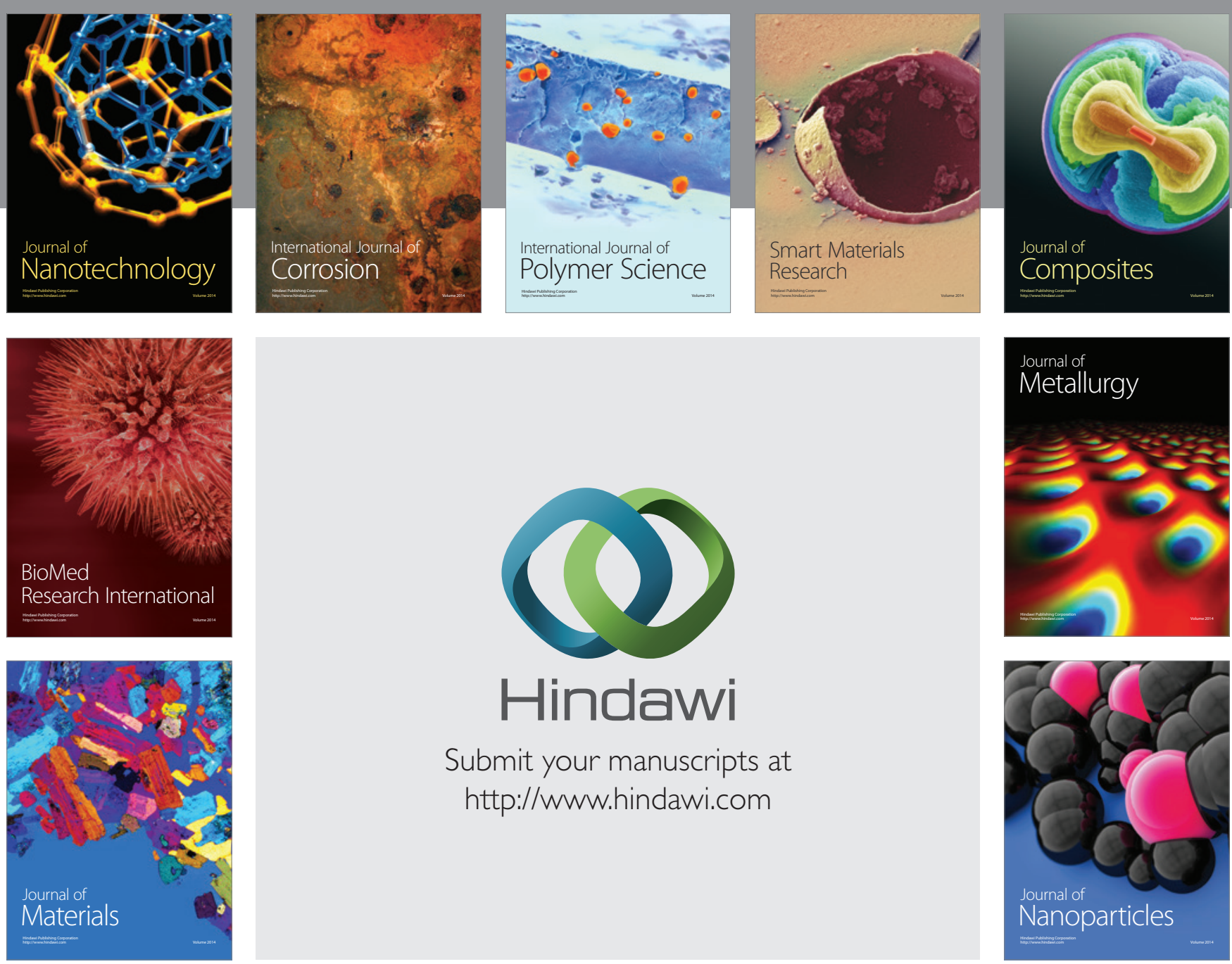

Submit your manuscripts at http://www.hindawi.com
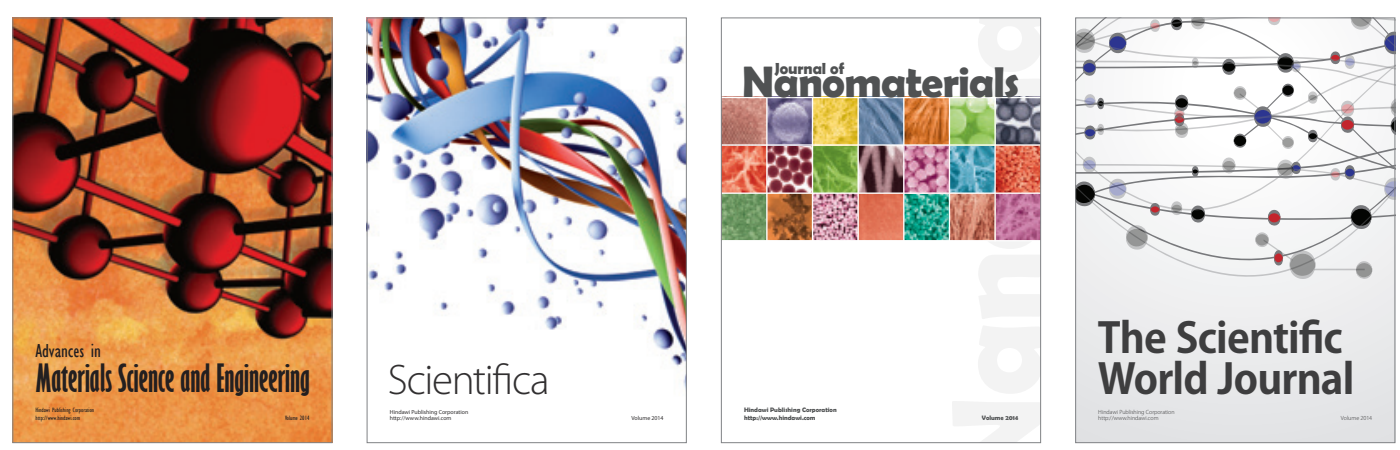

\section{The Scientific World Journal}
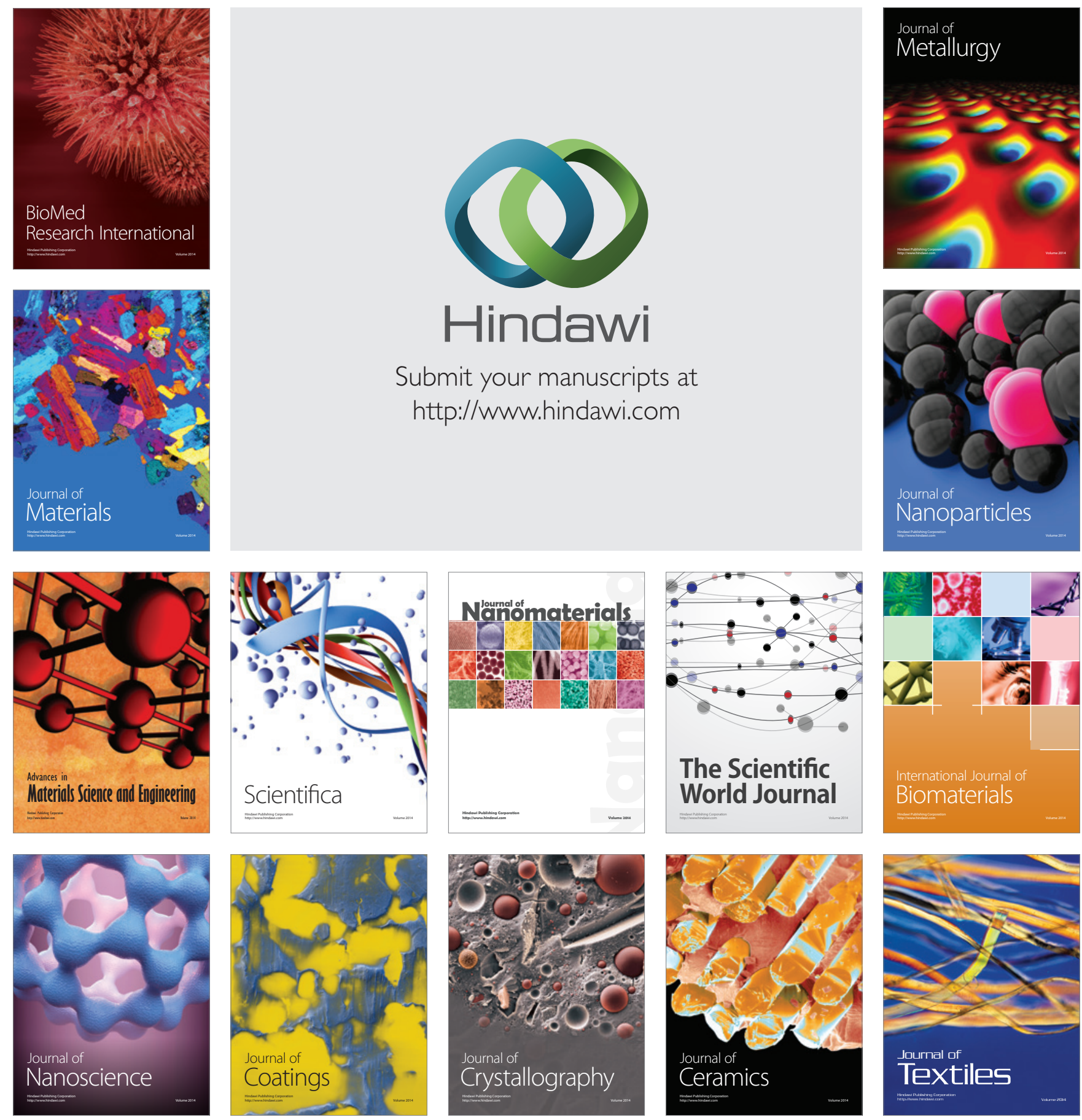